\title{
La educación para el emprendimiento como predictor de una intención emprendedora de estudiantes universitarios
}

\author{
Cecilia I. Vélez ${ }^{(1)}$, Miguel A. Bustamante ${ }^{(1,2) \star}$, Beatriz A. Loor ${ }^{(1)}$ y Sergio M. Afcha(1) \\ (1) Facultad de Especialidades Empresariales, Universidad Católica de Santiago de Guayaquil, Av. Carlos Julio \\ Arosemena Km. 11⁄2 vía Daule, Guayaquil, Ecuador. (correo-e: cecilia.velez@cu.ucsg.edu.ec, \\ beatriz.loor@cu.ucsg.edu.ec, sergio.afcha@uv.es) \\ (2) Facultad de Economía y Negocios, Universidad de Talca, Avenida Lircay S/N, Talca, Chile, Campus Lircay. Avenida \\ Lircay S/N, Talca, Chile. (correo-e: mabu@utalca.cl)
}

* Autor a quien debe ser dirigida la correspondencia.

Recibido Jul. 29, 2019; Aceptado Sep. 24, 2019; Versión final Nov. 19, 2019, Publicado Abr. 2020

\section{Resumen}

Se analiza el papel de la educación para el emprendimiento en la formación de intenciones emprendedoras de los estudiantes universitarios. Se analizó la conveniencia y la viabilidad percibida de la intención emprendedora considerando como variables independientes dos medidas alternativas de educación emprendedora. Para ello, se usó un modelo tipo probit para el análisis de variables dependientes discretas. Los análisis estadísticos se realizaron considerando una muestra de estudiantes de administración de empresa de cinco universidades de la ciudad de Guayaquil, Ecuador. Los resultados confirman el modelo de evento emprendedor mostrando que tanto viabilidad como conveniencia percibida tienen una influencia positiva y significativa sobre la intención emprendedora. Los resultados permiten afirmar que la educación emprendedora de por sí no tuvo una incidencia significativa sobre la intención emprendedora. La educación emprendedora sí resulta significativa porque permite mejorar competencias emprendedoras y potenciar algunas habilidades prácticas administrativas, el entendimiento de las actitudes, y los valores asociados al espíritu emprendedor.

\section{Education for entrepreneurship as a predictor of entrepreneurial intent of university students}

\begin{abstract}
The role of education for entrepreneurship in the formation of entrepreneurial intentions of university students is analyzed. The convenience and perceived viability of the entrepreneurial intention were analyzed considering two independent measures of entrepreneurial education as independent variables. For this, a probit model was used for the analysis of discrete dependent variables. Statistical analyzes were performed considering a sample of students of business administration from five universities in the city of Guayaquil, Ecuador. The results confirm the entrepreneurial event model showing that both viability and perceived convenience have a positive and significant influence on the entrepreneurial intention. The results allow us to affirm that entrepreneurial education in itself does not have a significant impact on entrepreneurial intent. Entrepreneurial education is significant because it allows improving entrepreneurial skills and enhancing some administrative practical skills, understanding attitudes, and the values associated with the entrepreneurial spirit.
\end{abstract}

Keywords: entrepreneurship; entrepreneurial intentions; entrepreneurial competences 


\section{INTRODUCCIÓN}

El emprendimiento desempeña un papel importante en el desarrollo económico en tanto estimula la incubación de innovaciones tecnológicas, el aumento de la eficiencia económica y la creación de nuevos empleos (Audretsch 2018). Es así como, durante las últimas décadas, el estudio de las actividades emprendedoras ha recibido atención académica y gubernamental en torno a los factores que determinan la intención emprendedora, IE (Douglas y Shepherd, 2002; Fernandez, 2018). En esta misma línea de análisis, se define la intencionalidad como un estado mental que dirige al individuo hacia un objetivo específico, en este caso, emprender un negocio y, por tanto, la intención emprendedora se analiza como una variable predictora del comportamiento planeado (Krueger, 1993) la cual está determinada por las actitudes personales y por las "influencias exógenas" de carácter situacional (Ajzen, 1991; Krueger et al., 2000). La intención emprendedora depende de los rasgos y personalidad del individuo incluyendo la autoeficacia (Zhao, 2005), la exposición a las actividades emprendedoras (Krueger 1993; Peterman y Kennedy, 2003) y el género (Gupta et al., 2008; Shinnar et al., 2012).

A nivel académico, existen varios modelos teóricos que permiten explorar la relación entre la IE y sus antecedentes. Después de una revisión de la literatura, se decidió basar la investigación en los modelos de Ajzen (1991) y de Shapero (1984), teniendo en consideración que estos dos modelos han sido validados por la literatura existente en numerosas ocasiones, ver por ejemplo Gird y Bagraim, (2008), Kautonen et al. (2015), Peterman y Kennedy, (2003) entre otros. El primer modelo es el de la teoría del comportamiento planificado introducido por Ajzen (1991) que consta de tres componentes que predicen la formación de la intención emprendedora, a saber 1) la actitud hacia el comportamiento, 2) normas subjetivas y 3) el grado de control del comportamiento percibido relacionado con la autoeficacia. Este modelo afirma que cualquier comportamiento requiere de un cierto nivel de planificación y, por tanto, pueden predecirse a partir de la intención de adoptar ese comportamiento.

La actitud emprendedora representa la valoración personal, positiva o negativa, que los individuos sienten hacia el emprendimiento (Ajzen, 1991). Es equivalente a la percepción de la deseabilidad personal en el modelo de Shapero (1982). Las normas subjetivas por su parte, miden la presión social percibida por parte de familiares, amigos u otras personas significativas (Ajzen, 1991) lo que incluye la expectativa de la familia sobre el comportamiento del individuo y el apoyo esperado de otras personas significativas, ante la posibilidad de que el individuo decida emprender su propio negocio. Sin embargo, este factor es difícil de capturar y tiene menos impacto predictivo para los sujetos que presentan un locus de control interno elevado o en aquellos individuos que manifiestan una fuerte orientación hacia la toma de decisiones y acciones. Con todo, diversos estudios han encontrado que no existe una relación directa y significativa entre las normas subjetivas y la IE (Krueger et al., 2000). Complementariamente, el control percibido, se refiere a la autopercepción de la capacidad de las personas para convertirse en empresarios; de manera similar, ha sido llamado autoeficacia y es equivalente a la viabilidad percibida en el modelo de Shapero y Sokol (1982).

El segundo modelo, el de Shapero y Sokol (1982) ha sido llamado también modelo de evento emprendedor, EEM, por sus siglas en inglés y se utiliza para describir un proceso empresarial donde la intencionalidad juega un rol central (Bird, 1988). Es por ello que este modelo considera la creación de empresas como un evento que puede explicarse mediante la interacción entre iniciativa, capacidad, gestión, autonomía relativa, y riesgo. El modelo, indica que la IE se deriva de las percepciones de viabilidad y conveniencia por parte del individuo, mismas que a su vez, establecen relaciones que se ven afectadas por el contexto cultural y social. Consecuente con lo indicado, esta percepción de la elección personal en entornos culturales y sociales ha sido analizada empíricamente por varios autores (Krueger et al. 2000, Peterman y Kennedy, 2003 y Wilson et al., 2007, entre otros). Al respecto Shapero y Sokol (1982) argumentan que la deseabilidad y la viabilidad percibidas, determinan la credibilidad relativa de los comportamientos alternativos y que la IE surge parcialmente de la exposición que tenga el individuo a la actividad empresarial.

Por otra parte, la educación emprendedora (Wilson et al. 2007), podría ser incluida en este modelo como un antecedente previo relevante que incide sobre la intención emprendedora (Gorman et al., 1997), puesto que proporciona habilidades (Bae, 2014) tales como la intuición, autoestima y competencias que permitan a la persona reconocer oportunidades. Así mismo, en general se reconoce el impacto de la educación emprendedora en el contexto de la formación universitaria, que resulta especialmente relevante cuando se analiza la percepción de los estudiantes respecto del emprendimiento (Peterman y Kennedy, 2003). Se valida, de esta forma el hecho que la percepción determina la intención emprendedora, misma que ha sido confirmada a nivel empírico (Byabashaija y Katono,2011) y que se traduce en cambios actitudinales relevantes (Ajzen,1991) que pueden ser desarrollados a través de la educación emprendedora como ocurre a nivel universitario.

La educación para el emprendimiento, y su ubicación en el ciclo de desarrollo del estudiante, así como su especificidad, puede tener un efecto directo sobre la intención emprendedora de las personas (Byabashaija y Katono, 2011), intención que a su vez puede verse influida por algunas variables moderadoras, tales como 
género, tipo de universidad, sea esta pública o privada, además del avance relativo del estudiante en su carrera (Byabashaijay Katono, 2011). Se conforma de esta forma, un sistema de factores que inciden de manera relevante sobre la intencion emprendedora (Soria-Barreto et al., 2016). Se hace necesario en consecuencia, poder realizar un diagnostico previo que permita determinar determinar si la educacion emprendedora contribuye de forma positiva sobre la intencion emprendedora, como lo afirman diferentes estudios (Lima et al., 2015; Zapkau et al., 2017; Fayolle y Gailly, 2013) que demuestran que los estudiantes universitarios efectivamente expresan sus aspiraciones de crear un negocio, sin embargo, al analizar actitudes y competencias se observan brechas que la educacion empresarial universitaria busca potenciar tranversalmente (Soria-Barreto, et al., 2016).

En general, la literatura confirma los postulados del análisis emprendedor por cuanto las percepciones de la deseabilidad y viabilidad percibidas conducen a la gestación de la capacidad de emprendimiento. Por una parte, se ratifican los hallazgos de Krueger (1993), puesto que ambas variables resultan ser inductoras significativas de la intención emprendedora (Ajzen 1991; Kautonen et al., 2015) y, por otra, que el emprendimiento puede ser aprendido (Gorman et al. 1997; Kuratko, 2003). A nivel secundario esta formación afecta positivamente la Intención Emprendedora (Peterman y Kennedy, 2003) y a nivel universitarios, la educación empresarial estimula la intención de iniciar un nuevo negocio (Wu y Wu, 2008).

Finalmente, considerando que los programas de apoyo a la iniciativa empresarial son efectivos a la hora de impulsal la conducta de los emprendedores (Kuratko 2005; Peterman y Kennedy 2003), se ratifica el hecho que la educación emprendedora promueve la intención emprendedora (Ajzen, 1991). En consecuencia y sobre la base de lo analizado hasta aquí, el presente trabajo pretende estudiar el papel de la educación para el emprendimiento en la formación de la intención emprendedoras de los estudiantes universitarios de entidades públicas y privadas de la ciudad de Guayaquil, Ecuador.

\section{METODOLOGÍA}

El presente trabajo es de carácter no experimental, descriptivo y de diseño transversal (Hernández, et al., 2014) siendo los datos recolectados mediante el método de encuesta, basado en el instrumento desarrollado por Liñán y Chen (2009) para validar el modelo de comportamiento planeado.

\section{Población y muestra}

Los datos examinados en este trabajo fueron obtenidos a partir de encuestas implementadas en cinco (5) universidades de la provincia de Guayaquil. A fin de obtener una muestra más o menos homogénea, se accedió a universidades categorizadas en el nivel de calidad B del Consejo de Evaluación, Acreditación y Aseguramiento de la Calidad de la Educación Superior del Ecuador CEAACES, que establece una clasificación de las universidades en cuatro categorías (A, B, C y D).

El trabajo de campo se realizó entre los meses de mayo y agosto de 2018 periodo en el cual se aplicaron 542 cuestionarios a una muestra aleatoria de estudiantes de las facultades de economía y administración de Empresas.

\section{Instrumento}

Entre los instrumentos utilizados para aplicar la teoría de la elección planeada de Azjen (1991) al ámbito de los estudios de emprendimiento se identificó el cuestionario de intención emprendedora propuesto y validado por Liñán y Chen (2009). A partir de los ítems que propone y siguiendo la literatura previa, se procedió a construir cuatro (4) escalas compuestas tipo Likert 1:7 puntos, conformadas por los ítems del instrumento, los cuales conforman las dimensiones Conveniencia percibida, Viabilidad percibida, Intención emprendedora y Papel de su universidad en materia de emprendimiento.

\section{Definición de las variables del estudio}

Se definió como variable dependiente Intención Emprendedora (IE) la cual, siguiendo el instrumento anterior, fue medida considerando 6 items valorados acorde con una escala tipo Likert $1: 7$ puntos, los cuales fueron promediados para obtener una escala compuesta. Se tuvo en consideración además que la variable IE es susceptible de tomar valores del 1 al 7, siendo 1 el nivel de intención emprendedora más bajo y 7 el más elevado. Una vez calculada esta escala, se procedió a construir una variable binaria de manera que, para valores iguales o por encima de 6, la intención emprendedora toma el valor 1, para valores por debajo de 6, la variable IE tomará el valor 0 . Esta recodificación, obedece a la necesidad de poder discriminar entre aquellos estudiantes realmente interesados en iniciar una actividad emprendedora y el resto de estudiantes. 
Como variables independientes se utilizaron las variables conveniencia percibida medida a través de una escala compuesta basada en la puntuación de los reactivos que la definen a partir de una escala tipo Likert 1 : 7 puntos, siendo 1 el valor más bajo de conveniencia percibida y 7 el más elevado. La segunda variable independiente fue viabilidad percibida (Krueger, 1993), también definida como una escala compuesta basada en la puntuación correspondiente a las preguntas que la conforman de manera similar a las dos escalas mencionadas anteriormente, esta dimensión fue valorada siguiendo una escala tipo Likert del 1: 7, siendo 1 el valor más bajo de viabilidad percibida y 7 el más elevado.

La tercera variable independiente fue experiencia empresarial y laboral (Gorman et al. 1997; Kuratko, 2005), que determina la exposición a la experiencia empresarial sobre la base de dos variables binarias tales como presencia de padre emprendedor, presencia de madre emprendedora además de la experiencia laboral del propio estudiante, todas ellas medidas en años de trabajo. Como cuarta variable independiente se determinó la educación emprendedora (Wu y Wu, 2008), misma que se midió de dos maneras. La primera de ellas, considera la realización de cursos de carácter obligatorio y optativos relacionados con el emprendimiento y que permite medir la exposición del estudiante a cursos específicos sobre emprendimiento. La segunda, tiene que ver con la contribución de dichos cursos para i) mejorar la capacidad del estudiante para identificar nuevas oportunidades de negocio, ii) acentuar sus habilidades prácticas administrativas y iii) potenciar las habilidades necesarias para identificar las actitudes y valores asociados a la conducta emprendedora (Peterman y Kennedy, 2003).

Finalmente, se incluyeron como variables de control, el sexo del estudiante (Shinnar et al. 2012) la edad del estudiante (Lévesque y Minniti, 2011) y una variable binaria indicando si el estudiante cursa su carrera en una universidad pública o privada (Pihie et al, 2013), teniendo en consideración que estas variables, han sido identificadas por la literatura como variables que pueden ejercer influencia sobre la intención emprendedora, motivo por el cual se incluyeron en la estimación.

\section{Criterios de modelamiento}

Dada la naturaleza binaria de la variable dependiente IE, se propuso un modelo de variable dependiente discreta, es decir, cuyo rango de valores se adecua a una escala binaria o dicotómica con dos valores posibles, hasta la policotómica con varios valores viables (Hernández, et al., 2014). En consecuencia, se aplicó un modelo de regresión probit que utiliza una función de distribución acumulativa normal para crear un modelo de probabilidad para una variable de respuesta binaria.

En este caso concreto, se busca conocer los factores que influyen sobre la probabilidad de que la IE para que ésta sea elevada. Finalmente, en consistencia con lo anterior, la ecuación que se desea estimar se muestra en (1).

$$
\operatorname{Pr}(I E=1)=\beta_{0}+\operatorname{Conv} \beta_{1_{+}} \operatorname{Via}_{2_{2}} \operatorname{Exp}_{\text {padre }} \beta_{3_{+}} \operatorname{Exp}_{\text {Madre } \beta_{4}}+\beta_{5} \operatorname{Exp}_{\text {lab }}+E E \beta_{6}+E d a d \beta_{7}+\operatorname{Masc} \beta_{\beta_{+}} P u b \beta_{9}+\varepsilon_{i}
$$

En esta ecuación: $E=$ intención emprendedora; Conv y Via= conveniencia y viabilidad percibida; Exppadre y Expmadre= la experiencia emprendedora del padre y la madre del individuo; EE= educación emprendedora; Explab = experiencia laboral del individuo; Edad = en años; Masc = Universidad pública; y Pub= variables de control edad, género y tipo de universidad.

\section{RESULTADOS}

La Tabla 1 muestra las estadísticas descriptivas de las variables incluidas en el modelo donde se observa que, en general, el número de contactos y entrevistados para la investigación fue el adecuado, alcanzando frecuencias entre 538 y 542 casos para cada una de las dimensiones analizadas. Las variables demográficas muestran que un $47 \%$ son hombres y $53 \%$ mujeres, con edades fluctúan entre 17 y 59 años, siendo el promedio de 23,65 años. Además, un $48 \%$ de los entrevistados estudia en una universidad pública, mientras que el $52 \%$ lo hace en universidades privadas. Mención especial merece uno de los casos entrevistados que corresponde a un adulto de 59 años que cursa uno de los programas de estudio abordados en esta investigación.

\section{Análisis descriptivo}

En general, se puede apreciar de la Tabla 1, que el 47\% de los encuestados tiene una elevada intención emprendedora; asimismo, la conveniencia percibida es más bien alta ubicándose por encima de los 5 puntos $(5,56)$ en una escala de rango $1-7$, lo cual permite interpretar que, para los estudiantes, el tema del emprendimiento, es valorado positivamente. Algo similar ocurre con la dimensión viabilidad percibida la que se ubica precisamente a nivel de 5 puntos con un rango de variabilidad de 1,8 puntos. 
Tabla 1: Estadística descriptiva

\begin{tabular}{|l|c|c|c|c|c|}
\hline Variable & Obs & Media & Desv. Est. & Min & Max \\
\hline Intención emprenedora & 542 & $47 \%$ & 0,50 & 0 & 1 \\
\hline Conveniencia percibida & 542 & 5,56 & 1,31 & 1 & 7 \\
\hline Viabilidad percibida & 542 & 5,00 & 1,85 & 1 & 7 \\
\hline Padre Emprendedor & 538 & $17 \%$ & 0,38 & 0 & 1 \\
\hline Madre Emprendedora & 538 & $11 \%$ & 0,31 & 0 & 1 \\
\hline Experiencia laboral & 542 & 2,73 & 4,86 & 0 & 45 \\
\hline $\begin{array}{l}\text { Educación } \\
\text { emprendedora_1 }\end{array}$ & 542 & $40 \%$ & 0,49 & 0 & 1 \\
\hline $\begin{array}{l}\text { Educación } \\
\text { emprendedora_2 }\end{array}$ & 542 & 4,95 & 1,58 & 1 & 7 \\
\hline Sexo (masculino=1) & 542 & $47 \%$ & 0,50 & 0 & 1 \\
\hline Edad & 542 & 23,65 & 5,50 & 17 & 59 \\
\hline Universidad pública & 542 & $48 \%$ & 0,50 & 0 & 1 \\
\hline
\end{tabular}

Respecto a los padres, en general, los datos expresan que éstos presentan escasa experiencia emprendedora, sólo el $17 \%$ de estudiantes tiene padres que trabajan por cuenta propia frente a un $11 \%$ en el caso de las madres. Finalmente, los encuestados manifiestan, en promedio, tener una experiencia laboral de 2,73 años, aunque esta presenta una gran variabilidad alcanzando una desviación estándar de 4,83, siendo 0 el mínimo y 45 al máximo de años trabajados por los estudiantes entrevistados. En relación con la educación emprendedora, como ya se mencionó, se miden dos variables. Por una parte, el $40 \%$ afirmó haber realizado algún curso relacionado con esta materia (educación emprendedora_1), mientras que, respecto a la variable educación emprendedora_2, los estudiantes muestran una percepción elevada $(4,95)$ respecto a la contribución de los cursos al emprendimiento sobre las diferentes competencias que componen esta variable.

Complementariamente, las tablas 2 y 3 , muestran los resultados de las estimaciones probit, las cuales permiten identificar aquellas variables que influyen sobre la probabilidad de IE considerando un nivel de significación al $1 \%\left(^{* * *} ; p<0,01\right)$. Se aprecia que el modelo 1 de la Tabla 2 , representa el modelo básico, en el cual únicamente están incluidas las variables dependientes, confirmando la influencia de la conveniencia percibida $\left({ }^{* * *}\right)$ y la viabilidad percibida ${ }^{* * *}$ ) sobre la intención emprendedora. Por otra parte, respecto de la conveniencia percibida se aprecia que los valores para la conveniencia muestran que el cambio de una (1) unidad en el nivel de conveniencia percibida incrementa en 13,1 puntos porcentuales la probabilidad de que la IE sea elevada.

Así mismo, en el caso de la viabilidad percibida, este valor es ligeramente menor, mostrando que el cambio de un punto (1) en la viabilidad percibida, incrementa en unos 12,1 puntos porcentuales la probabilidad de que la IE sea elevada. Por otra parte, el resto de las variables resultaron no significativas, mostrando escasa influencia sobre la IE, lo cual permite indicar que la influencia de la experiencia emprendedora y la mera realización de cursos de emprendimientos (educación emprendedora_1) presentan escasa incidencia sobre la intención emprendedora. Por otro lado, estudios previos ratifican estos resultados donde se indica que los estudiantes que ejecutan y crean negocios tiene un índice de actitud emprendedora potencial similar a los universitarios que no lo hacen, sin embargo, los resultados del estudio de Soria-Barreto et al., (2016) manifiesta que los universitarios con educación empresarial tienen una mayor probabilidad de crear emprendimientos exitosos.

Adicionalmente y desde una perspectiva general, la educación emprendedora EE que analiza la pertenencia a una universidad pública, sexo y estar cursando final de carrera, aunque muestra incidencia en los modelos 3,4 y 5 , no resulta significativa. Seguidamente, para validar el papel de la educación emprendedora de una manera alternativa, se incorporó la variable EE2, medida en términos cuadráticos ( $\left.{ }^{*} 2\right)$ captura si la variable sigue una distribución cuadrática en forma de "U" o invertida, la cual permite examinar si aquellos alumnos que realizaron cursos de educación emprendedora que refuerzan sus habilidades como potenciales emprendedores, generan una influencia sobre la IE. Al respecto, los resultados, mostrados en la Tabla 3, que presenta la medición de cada variable, muestra en la parte inferior de cada índice la desviación estándar entre paréntesis, lo cual permite confirman la importancia relativa de la educación emprendedora sobre la IE. 
Tabla 2: Modelo probit utilizando las variables dependientes

\begin{tabular}{|c|c|c|c|c|c|}
\hline & Modelo 1 & Modelo 2 & Modelo 3 & Modelo 4 & Modelo 5 \\
\hline $\operatorname{Pr}(\mathrm{IE}=1)$ & $d y / d x$ & $d y / d x$ & $d y / d x$ & $d y / d x$ & $\mathrm{dy} / \mathrm{dx}$ \\
\hline \multirow[t]{2}{*}{ Conveniencia percibida } & $0,131^{* \star *}$ & $0,130^{\star * \star}$ & $0,132^{* \star *}$ & $0,132^{\star \star \star}$ & $0,131^{\star \star \star}$ \\
\hline & $(0,01)$ & $(0,02)$ & $(0,01)$ & $(0,01)$ & $(0,01)$ \\
\hline \multirow[t]{2}{*}{ Viabilidad percibida } & $0,121^{\star \star \star}$ & $0,121^{\star \star \star}$ & $0,126^{\star \star \star}$ & $0,126^{\star * \star}$ & $0,124^{\star \star \star}$ \\
\hline & $(0,02)$ & $(0,02)$ & $(0,01)$ & $(0,02)$ & $(0,01)$ \\
\hline \multirow[t]{2}{*}{ Padre Emprendedor } & $-0,026$ & $-0,024$ & $-0,026$ & $-0,026$ & $-0,024$ \\
\hline & $(0,04)$ & $(0,04)$ & $(0,04)$ & $(0,04)$ & $(0,04)$ \\
\hline \multirow[t]{2}{*}{ Madre Emprendedora } & $-0,074$ & $-0,069$ & $-0,07$ & $-0,070$ & $-0,068$ \\
\hline & $(0,04)$ & $(0,05)$ & $(0,04)$ & $(0,04)$ & $(0,04)$ \\
\hline \multirow[t]{2}{*}{ Experiencia laboral } & 0,000 & $-0,002$ & $-0,002$ & $-0,002$ & $-0,003$ \\
\hline & $(0,00)$ & $(0,01)$ & $(0,01)$ & $(0,01)$ & $(0,01)$ \\
\hline \multirow[t]{2}{*}{ EE } & 0,033 & 0,029 & & & \\
\hline & $(0,03)$ & $(0,03)$ & & & \\
\hline \multirow[t]{2}{*}{ Sexo (Masculino=1) } & & 0,012 & & & \\
\hline & & $(0,03)$ & & & \\
\hline \multirow[t]{2}{*}{ Edad } & & 0,015 & 0,016 & 0,016 & 0,014 \\
\hline & & $(0,01)$ & $(0,01)$ & $(0,01)$ & $(0,01)$ \\
\hline \multirow[t]{2}{*}{ Edad^2 $^{\wedge}$} & & $-0,00$ & $-0,000$ & $-0,000$ & $-0,000$ \\
\hline & & $(0,00)$ & $(0,00)$ & $(0,00)$ & $(0,00)$ \\
\hline \multirow[t]{2}{*}{ Universidad Pública } & & 0,001 & & & \\
\hline & & $(0,03)$ & & & \\
\hline \multirow[t]{2}{*}{ EE*Universidad Pública } & & & 0,001 & & \\
\hline & & & $(0,05)$ & & \\
\hline \multirow[t]{2}{*}{ EE *sexo } & & & & 0,008 & \\
\hline & & & & $(0,04)$ & \\
\hline \multirow[t]{2}{*}{ EE*Final de carrera } & & & & & 0,101 \\
\hline & & & & & $(0,09)$ \\
\hline № de observaciones & 538 & 538 & 538 & 538 & 538 \\
\hline
\end{tabular}

Tabla 3: Modelo probit confirmatorio de la intención emprendedora

\begin{tabular}{|l|c|c|}
\hline & Modelo 1 & Modelo 2 \\
\hline $\operatorname{Pr}(\mathrm{IE}=1)$ & & \\
\hline Conveniencia percibida & $0,129^{\star \star \star}$ & $0,128^{\star \star *}$ \\
\hline & $(0,01)$ & $(0,01)$ \\
\hline Viabilidad percibida & $0,109^{\star \star \star}$ & $0,110^{\star * *}$ \\
\hline & $(0,02)$ & $(0,02)$ \\
\hline Padre Emprendedor & $-0,025$ & $-0,019$ \\
\hline & $(0,04)$ & $(0,04)$ \\
\hline Madre Emprendedora & $-0,082$ & $-0,074$ \\
\hline & $(0,04)$ & $(0,04)$ \\
\hline Experiencia laboral & 0,000 & $-0,001$ \\
\hline & $(0,00)$ & $(0,01)$ \\
\hline EE2 & $0,032^{\star *}$ & $0,035^{\star * *}$ \\
\hline & $(0,01)$ & $(0,01)$ \\
\hline Sexo (Masculino=1) & & 0,010 \\
\hline & & $(0,03)$ \\
\hline Edad & & 0.017 \\
\hline & & $(0,01)$ \\
\hline Edad^2 & & $-0,000$ \\
\hline & & $(0,00)$ \\
\hline Universidad Pública & & 0,024 \\
\hline & & $(0.04)$ \\
\hline № de observaciones & 538 & 538 \\
\hline
\end{tabular}


En general, se aprecia que los resultados de los modelos 1 y 2 de la Tabla 3 , son bastante similares ya que, nuevamente las variables de conveniencia percibida y viabilidad percibida resultaron significativas y con efectos marginales prácticamente idénticos al modelo básico mientras que el resto de variables resultaron igualmente no significativas. Por otra parte, se observa en particular que la variable conveniencia percibida alcanza índices relevantes de incidencia $\left(0,128^{* * *}\right)$ con alta significación al $1 \%$ y que las variables de viabilidad percibida entregaron índices también significativos $\left(0,110^{\star \star \star}\right)$ confirmando finalmente que la educación en emprendimiento, sigue siendo significativa.

Comparando los resultados obtenidos respecto a las variables de educación emprendedora de las tablas 2 y 3 , se puede afirmar por tanto, que el hecho de participar de cursos de educación emprendedora mejoran la IE únicamente en la medida que estos cursos contribuyen positivamente a reforzar las habilidades y competencias emprendedoras de los estudiantes. En consecuencia, sobre la base de lo señalado y en la medida que la variable de educación emprendedora EE2 está compuesta por sub-items que capturan el impacto de la educación para el emprendimiento sobre una serie de competencias y valores favorables hacia la actividad emprendedora, podría afirmarse que los cursos de emprendimiento favorecen la intención emprendedora y posibilitan a los estudiantes a crear emprendimientos exitosos.

Finalmente, del análisis de las variables demográficas de control Sexo (Masculino=1), Edad y pertenencia a una Universidad Pública, no mostraron incidencia significativa en ninguno de los modelos analizados, así como tampoco fueron significativas en ninguna de sus interacciones con la variable de educación para el emprendimiento.

\section{DISCUSIÓN}

Los coeficientes determinados muestran los efectos marginales positivos y significativos al $1 \%$ de las variables independientes de conveniencia percibida $\left(0,129^{* * *} ; 0,128^{* * *}\right)$ y viabilidad percibida $\left(0,109^{* * *} ; 0,110^{* * *}\right)$, como expresiones de rasgos y personalidad de los individuos (Zhao et al., 2005) sobre la variable dependiente intención emprendedora (Bae, 2014; Gorman et al., 1997; Liñan et al., 2009), confirmando el hecho que un cambio en las variables independientes provoca un cambio efectivo de la variable dependiente, sin perjuicio que se mantienen constantes el resto de las variables.

Concordante con lo indicado y como se puede observar del modelo 1 analizado, este primer modelo confirma la influencia de la conveniencia percibida y la viabilidad percibida sobre las actividades emprendedoras (Krueger 1993; Peterman y Kennedy, 2003), resultado que se alinea con otros trabajos que demuestran la relevancia de la teoría que describe la intención emprendedora en el contexto el universitario y que confirman cómo la educación emprendedora puede tener un efecto directo sobre la IE (Peterman y Kennedy, 2003) cuando se incorpora la variable EE2, la cual permite examinar, a nivel empírico (Byabashaija y Katono, 2011), si los alumnos que realizan cursos de educación emprendedora refuerzan sus habilidades y acentúan actitudes emprendedoras (Ajzen,1991) demostrando que efectivamente, la educación emprendedora tiene influencia sobre la IE (Byabashaija y Katono, 2011).

Por otra parte, los resultados permiten confirmar los planteamientos propuestos en el marco conceptual (Douglas y Shepherd, 2002; Fernandez, 2018) según el cual el modelo de evento emprendedor alcanza resultados similares al de Azjen (1991) quien plantea que las acciones vienen precedidas por una intención planeada (Byabashaija y Katono, 2011). Sin embargo, la decisión de emprender no ocurre de manera espontánea, sino que está influida por diferentes dimensiones externas (Ajzen, 1991; Krueger et al., 2000) las cuales pueden de manera gradual y eventualmente influir en la formación de expectativas y percepciones del individuo sobre la acción que planea emprender, a saber, la conveniencia y la viabilidad percibida (Ajzen, 1991).

Respecto del rol de los padres se observa que en el caso de Madre Emprendedora, los índices son negativos para los modelos 1 y $2(-0,082$ y $-0,074)$, del mismo modo en el caso de Padre Emprendedor, los índices resultan igualmente negativos $(-0,025$ y $-0,019)$ para los modelo 1 y 2 , en ambos casos no significativos, en consecuencia, esta variable no permite explicar la intención emprendedora de los estudiantes analizados.

En general, los resultados obtenidos permitieron confirmar resultados similares a los hallados en la literatura previa de IE, no obstante, se rechaza la influencia de la experiencia emprendedora, sobre la intención emprender de los estudiantes universitarios (Peterman y Kennedy, 2003). Aunque con un impacto modesto, se confirma que la educación emprendedora incide sobre la intención emprendedora (Ajzen, 1991), ratificando el hecho que, la opción de los estudiantes de participar de cursos de EE efectivamente estimula su conducta, por una parte, porque el emprendimiento puede ser aprendido (Gorman et al., 1997; Kuratko, 2003) y, por otra, porque en la medida que los contenidos y las metodologías efectivamente permiten reforzar habilidades y competencias, la intención emprendedora se estimula positivamente y, aunque, el emprendimiento puede 
ser adquirido, puede ser progresivamente desarrollado a través de la educación emprendedora (Peterman y Kennedy, 2003). Todo lo cual ha sido evidenciado en este trabajo a nivel universitario (Wu y Wu, 2008). Así mismo, las variables independientes conveniencia percibida y viabilidad percibida (Krueger 1993; Peterman y Kennedy, 2003) inciden significativamente y las variables de control edad, sexo y tipo de universidad (Byabashaijay Katono, 2011) no inciden significativamente sobre la variable dependiente intención emprendedora.

\section{CONCLUSIONES}

Luego del análisis de las variables predictoras de la intención de emprendimiento de los estudiantes universitarios presentados en este trabajo se puede concluir que existe una elevada intención emprendedora, misma que se manifiesta en una relativamente alta conveniencia percibida y alta viabilidad percibida confirmando que los estudiantes valoran el emprendimiento.

Se concluye que los padres presentan escasa experiencia emprendedora y que los estudiantes muestran escasa experiencia laboral, sin embargo, han participado en cursos de emprendimiento y aprecian la contribución de estos a sus competencias emprendedoras, en consecuencia, la influencia de la experiencia emprendedora y la mera realización de cursos de emprendimientos presentan escasa incidencia sobre la intención emprendedora.

Entre las variables que inciden en la intención emprendedora de los estudiantes se concluye que conveniencia percibida incide en alto grado en tanto que viabilidad percibida lo hace pero en un grado levemente inferior, ambas en forma significativa. Sin embargo, las demás variables tales como experiencia emprendedora, educación emprendedora, pertenencia a una universidad pública, sexo y estar cursando final de la carrera, muestran incidencia relativamente baja pero resultaron no significativas.

El análisis de las variables de educación emprendedora permite afirmar que el hecho de participar de cursos de educación emprendedora mejoran la intención emprendedora en la medida que estos cursos relevan valores favorables a la actividad de emprender y en consecuencia contribuyen positivamente a reforzar las habilidades y competencias emprendedoras de los estudiantes.

\section{REFERENCIAS}

Ajzen, I., The Theory of Planned Behavior. https://doi.org/10.1016/0749-5978(91)90020-T Organizational Behavior and Human Decision Processes, 50(2), 179-211 (1991).

Audretsch, D. B., Entrepreneurship, Economic Growth, and Geography, https://doi.org/10.1093/oxrep/gry011 Oxford Review of Economic Policy 34(4) 637-651 (2018).

Bae, T. J., Qian, S., Miao, C. y Fiet, J. O., The Relationship Between Entrepreneurship Education and Entrepreneurial Intentions: A Meta-Analytic Review. https://doi.org/10.1111/etap.12095 Entrepreneurship theory and practice 38 (2) 217254 (2014).

Bird, B., Implementing entrepreneurial ideas: The case for Intention. Academy of Management Review, 13(3), 442-453 (1998).

Byabashaija, W. y Katono, I., The Impact of College Entrepreneurial Education on Entrepreneurial Attitudes And Intention To Start A Business In Uganda. https://doi.org/10.1142/S1084946711001768 Journal Of Developmental Entrepreneurship, 16(01), 127-144 (2011).

Douglas, E. y Shepherd, D. A., Self-Employment as a Career Choice: Attitudes, Entrepreneurial Intentions, and Utility Maximization. Entrepreneurship theory and practice 26(3) 81-90 (2002).

Fayolle, A. y Gailly, B., The Impact of Entrepreneurship Education on Entrepreneurial Attitudes and Intentions: Hysteresis and Persistence. https://doi.org/10.1111/jsbm.12065 Journal of Small Business Management (2013).

Fernandes, C., Ferreira, J. J., Raposo, M., Sanchez, J. y Hernandez-Sanchez, B., Determinants of Entrepreneurial Intentions: an International Cross-Border Study. https://doi.org/10.1108/IJIS-02-2017-0017 International Journal of Innovation Science 10(2) 129-142 (2018).

Gird, A. y Bagraim, J. J., The Theory of Planned Behaviour as Predictor of Entrepreneurial Intent Amongst Final-Year University Students. https://doi.org/10.1177/008124630803800410 South African Journal of Psychology, 38(4), 711-724 (2008).

Gorman, G., Hanlon, D. y King, W., Some Research Perspectives on Entrepreneurship Education, Enterprise Education and Education for Small Business Management: a Ten-Year Literature Review. International small business journal, 15(3), 56-78 (1997)

Gupta, V. K., Turban, D. B. y Bhawe, N. M., The Effect of Gender Stereotype Activation on Entrepreneurial Intentions. https://doi.org/10.1037/0021-9010.93.5.1053 Journal of Applied Psychology, 93(5), 1053 (2008). 
Hernández, S. R., Fernández, C. C., y Baptista, L. P., Metodología de la Investigación. Sexta Edición. Editorial Mc Graw Hill. México (2014).

Kautonen, T., Van Gelderen, M. y Fink, M., Robustness of the Theory of Planned Behavior in Predicting Entrepreneurial Intentions and Actions. https://doi.org/10.1111/etap.12056 Entrepreneurship Theory and Practice, 39(3), 655-674 (2015).

Krueger, N. F., Reilly, M. D. y Carsrud, A. L., Competing Models of Entrepreneurial Intentions. https://doi.org/10.1016/S0883-9026(98)00033-0 Journal of Business Venturing, 15(5-6), 411-432 (2000).

Krueger, N. F. The Impact of Prior Entrepreneurial Exposure on Perceptions of New Venture Feasibility and Desirability. https://doi.org/10.1177/104225879301800101 Entrepreneurship Theory and Practice, 18(1), 5-21 (1993).

Kuratko, D. F., The Emergence of Entrepreneurship Education: Development, Trends, and Challenges. https://doi.org/10.1111/j.1540-6520.2005.00099.x Entrepreneurship Theory and Practice, 29(5), 577-597 (2005).

Kuratko, D. F., Entrepreneurship education: Emerging Trends and Challenges for the 21st Century. White Paper, US Association of Small Business Education, 22, (2003).

Lévesque, M. y Minniti, M., Age matters: How Demographics Influence Aggregate Entrepreneurship. https://doi.org/10.1002/sej.117 Strategic Entrepreneurship Journal, 5(3), 269-284 (2011).

Lima E., Lopes, R. M., Nassif, V. y Da Silva, D., Opportunities to Improve Entrepreneurship Education: Contributions Considering Brazilian Challenges. https://doi.org/10.1111/jsbm.12110 Journal of Small Business Management, 53(4), 1033-1051 (2015).

Liñán, F. y Chen, Y-W, Development and Cross-Cultural Application of a Specific Instrument to Measure Entrepreneurial Intentions. https://doi.org/10.1111/j.1540-6520.2009.00318.x Entrepreneurship Theory and Practice, 33(3), 593-617 (2009)

Peterman, N. y Kennedy, J., Enterprise Education: Influencing Students' Perceptions of Entrepreneurship. https://doi.org/10.1046/j.1540-6520.2003.00035.x Entrepreneurship Theory and Practice 28(2) 129-144 (2003).

Zaidatol, A. L. P., Bagheri, A., Abdullah, S. Z. H., Knowledge of Cognition and Entrepreneurial Intentions: Implications for Learning Entrepreneurship in Public and Private Universities. Procedia-Social and Behavioral Sciences, 97, 174-181 (2013)

Shapero, A., The Entrepreneurial Event. In: Kent C.A. (ed.) The Enviroment for Entrepreneurship. Lexington Book. Toronto 21-40 (1984).

Shapero, A. y Sokol, L., The social dimensions of entrepreneurship. Encyclopedia of entrepreneurship, 72-90 (1982).

Shinnar, R. S., Giacomin, O. y Janssen, F., Entrepreneurial Perceptions and Intentions: The Role of Gender and Culture. https://doi.org/10.1111/j.1540-6520.2012.00509.x Entrepreneurship Theory and Practice 36(3) 465-493 (2012).

Soria-Barreto, K., Zuniga-Jara, S., y Ruiz-Campo, S., Educación e Intención Emprendedora en Estudiantes Universitarios: Un Caso de Estudio. http://dx.doi.org/10.4067/S0718-50062016000100004 Formación Universitaria, 9(1), 25-34. (2016)

Wilson, F., Kickul, J. y Marlino, D., Gender, Entrepreneurial Self-Efficacy, and Entrepreneurial Career Intentions: Implications for Entrepreneurship Education. https://doi.org/10.1111/j.1540-6520.2007.00179.x Entrepreneurship theory and practice, 31(3), 387-406 (2007).

Wu, S. y Wu, L., The impact of Higher Education on Entrepreneurial Intentions of University Students in China. https://doi.org/10.1108/14626000810917843 Journal of Small Business and Enterprise Development, 15(4), 752-774 (2008).

Zapkau, F. B., Schwens, C. y Kabst, R., The role of prior entrepreneurial exposure in the entrepreneurial process: a review and future research implications. https://doi.org/10.1111/jsbm.12232 Journal of Small Business Management, 55(1), 5686 (2017).

Zhao F., Exploring the Synergy Between Entrepreneurship and Innovation. https://doi.org/10.1108/13552550510580825 International Journal of Entrepreneurial Behavior \& Research, 11(1), 25-41 (2005). 
